\title{
Waveguide coupled MSM photodiode for surface plasmon polariton detection
}

\author{
E. Panchenko, T.D. James, and A. Roberts, School of Physics, University of Melbourne
}

Abstract - The requirement of in-plane detection is crucial for large scale plasmonic technology. Metal-semiconductormetal photodiodes can be used as ultrafast detectors in plasmonic integrated circuits. In this paper we show numerical simulations of the MSM photodiode coupled to a plasmonic waveguide.

Index Terms - optical waveguides, photodetectors, plasmons, semiconductor devices.

\section{INTRODUCTION}

Metal-semiconductor-metal (MSM) photodiodes are commonly used in ultrafast photoelectronic devices. Recently it was shown $[1,2]$ that surface plasmons can sufficiently enhance photodetector sensitivity at both infrared and visible wavelengths. Such structures are of great interest for plasmonics since they can be used for fast, broadband detection. In most cases top-down illumination is used and enhancement is produced by excitation of localised surface plasmons (LSP). Here we propose a MSM photodiode coupled with a plasmonic waveguide (see Fig. 1) suitable for in-plane surface plasmon polariton (SPP) detection. Such a setup could be used as a planar detector in plasmonic integrated circuits.

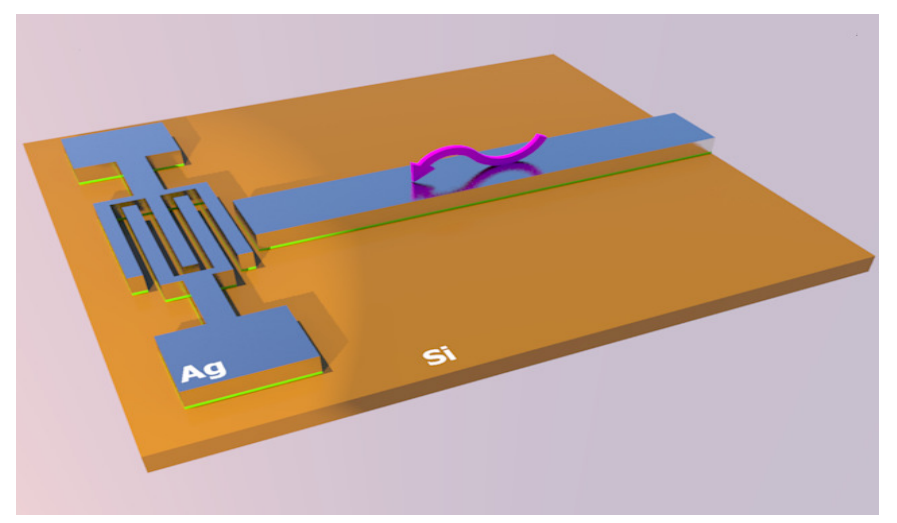

Fig. 1. Schematic representation of a plasmonic waveguide coupled with a MSM photodiode. The plasmonic waveguide and photodiode's fingers are 60 $\mathrm{nm}$ thick silver (grey). A titanium layer (green) of $2 \mathrm{~nm}$ thickness is simultaneously used as an adhesion layer and to form a Schottky barrier between the silver and silicon (yellow) substrate.

E. Panchenko is with the School of Physics, University of Melbourne, Victoria 3010, Australia (e-mail: epanchenko@student.unimelb.edu.au).

T.D. James is with the School of Physics, University of Melbourne, Victoria 3010, Australia (e-mail: timothy.james@unimelb.edu.au).

A. Roberts is with the School of Physics, University of Melbourne, Victoria 3010, Australia (e-mail: ann.roberts@unimelb.edu.au).

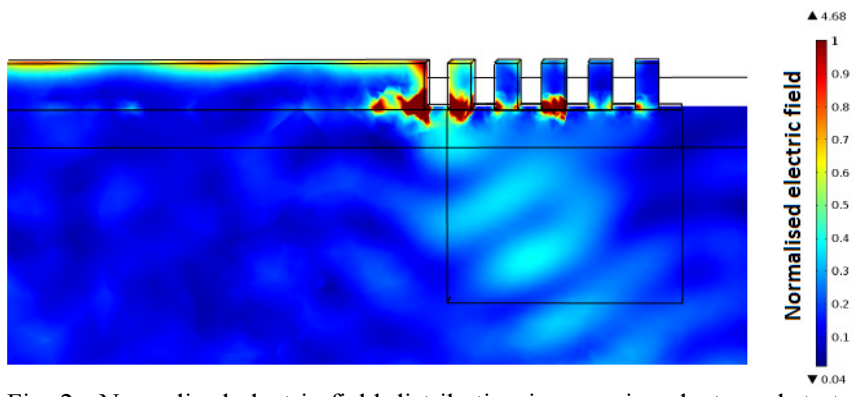

Fig. 2. Normalised electric field distribution in a semiconductor substrate A surface plasmon is decoupled from a waveguide into the active zone under the fingers of the photodiode.

\section{PRINCIPALS OF OPERATION}

A plasmonic port [3] was used to excite surface plasmons and guide them to an active zone of the MSM photodiode. An insulator-metal-insulator waveguide is coupled to the fingers of the photodiode. Several phenomena are utilized in the operation of this device. The metal fingers act as nanoantennas, enhancing the electric field intensity in the active region of photodiode. In addition, these fingers couple SPPs into the far-field, allowing photons to penetrate into the semiconductor substrate (see Fig. 2).

The design of the MSM photodiode consists of two electrodes which form Schottky junctions with the semiconductor substrate. These junctions are biased in opposing directions, forming an internal electric field within the semiconductor substrate. Photons absorbed in this region will excite an electron-hole pair which will be swept by the internal electric field between the electrodes, creating a photocurrent.

\section{MODELING}

In this work we used a $300 \mathrm{~nm}$ wide silver waveguide coupled to the MSM photodiode and varied the period of the fingers. Surface plasmon excitation at a free-space wavelength of $632.8 \mathrm{~nm}$ used. Despite the fact that both hotelectron injection through the Schottky barrier and electronhole generation phenomena are taking place the latter effect dominates at this wavelength. Therefore, assuming that all absorbed photons generate an electron-hole pair, we calculated the generation rate spatial distribution via [4]:

$$
\Gamma(r, \omega)=-(\pi / h) \operatorname{Im}(\varepsilon(r, \omega))|E(r, \omega)|^{2}
$$

where $E$ is an electric field in active region and $\operatorname{Im}(\varepsilon)$ is an imaginary part of silicon dielectric permittivity. 

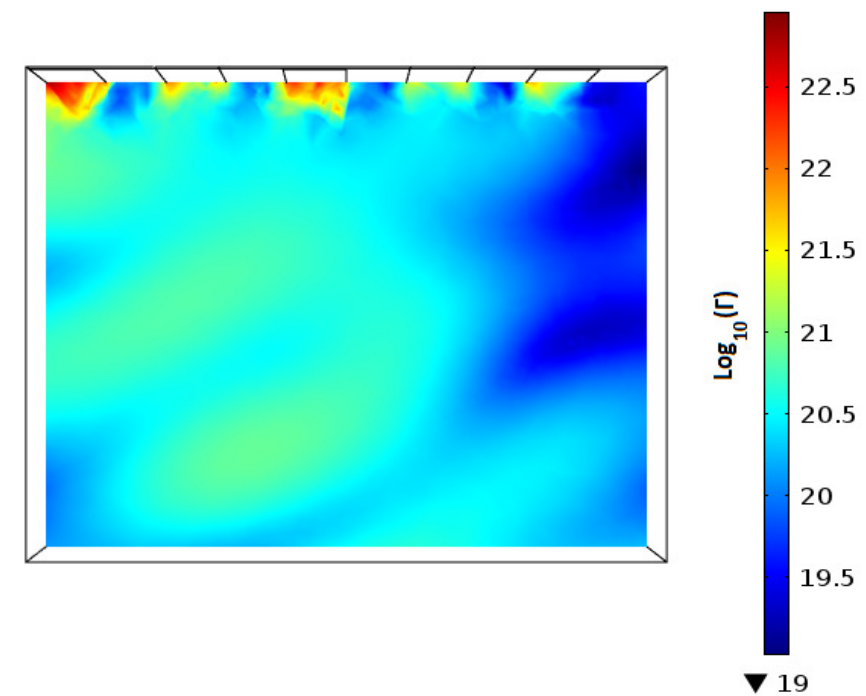

Fig. 3. Electron-hole generation rate profile under the photodiode's fingers (logarithmic scale). Note the increasing of the generation rate under the metal fingers which is produced by localized surface plasmon field enhancement.

Fig. 3 shows that the generation rate beneath the fingers of the photodiode is increased relative to the other areas due to the electric field enhancement produced by the localised surface plasmon excited on the fingers. A similar result was previously observed using top-down illumination [1]. This also leads to the enhancement of hot-electron injection through metal-semiconductor contact and can be used for near infrared detection.

The relation between irradiance delivered to the photodiode's active region and the periodicity of its fingers was also investigated. Fig. 4 shows that the irradiance delivered to this region is maximal at $60 \mathrm{~nm}$ and reduces with increasing period. The increasing period increases the separation of the fingers, weakening the LSP resonance by reducing the near-field coupling of each finger with its neighbors.

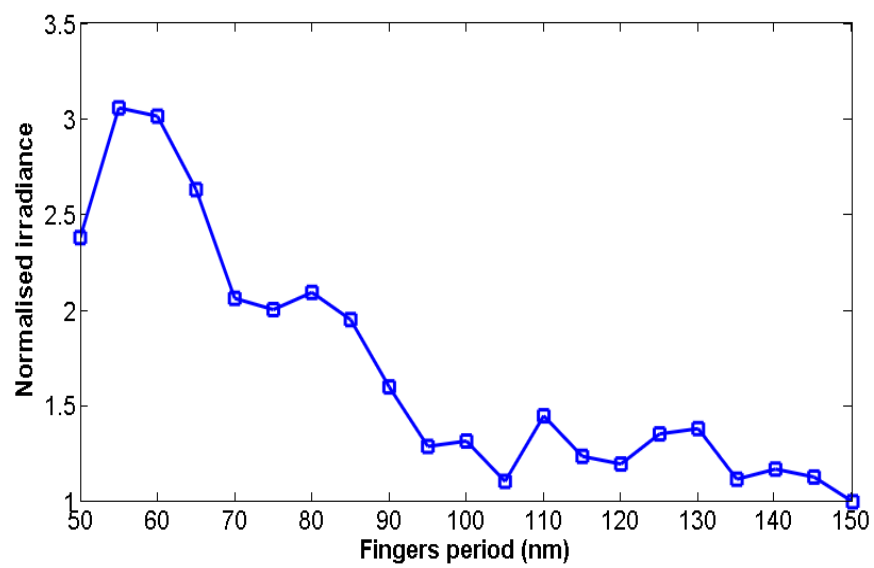

Fig. 4. Dependence of irradiance in the photodiode's active zone and period of the fingers. The $y$-axis is normalized to the irradiance at a finger period of $150 \mathrm{~nm}$.

\section{CONCLUSION}

We have simulated the electron-hole pair generation rate in the active region of a MSM photodiode coupled with a plasmonic waveguide. Such a design provides ability for planar integration and is fully CMOS compatible. Also it is much easier to fabricate in comparison to the design suggested by other groups.

Future investigations will involve fabrication and experimental measurements of responsivity as well as speed characteristics of the proposed device.

\section{ACKNOWLEDGMENT}

This research was supported under the Australian Research Council's Discovery Projects funding scheme (project number DP110100221). This work was performed in part at the Melbourne Centre for Nanofabrication (MCN) in the Victorian Node of the Australian National Fabrication Facility (ANFF).

\section{REFERENCES}

[1] Ali Sobhani, Mark W Knight, Yumin Wang, Bob Zheng, Nicholas S King, Lisa V Brown, Zheyu Fang, Peter Nordlander, and Naomi J Halas. Narrowband photodetection in the near-infrared with a plasmoninduced hot electron device. Nature communications, 4:1643, 2013.

[2] Hetterich, J., et al. "Optimized design of plasmonic MSM photodetector."Quantum Electronics, IEEE Journal of 43.10 (2007): 855-859.

[3] E. Panchenko, T.D. James and A. Roberts. Design of input port for plasmonic integrated circuits. Paper presented at: ICONN 2014, International Conference on Nanoscience and Nanotechnology, 2014 February 2-6, Adelaide, Australia.

[4] Mousavi, Saba Siadat, Andreas Stöhr, and Pierre Berini. "Plasmonic photodetector with terahertz electrical bandwidth." Applied Physics Letters 104.14 (2014): 143112. 